\title{
Recomendação de calagem a taxa variada sob diferentes intensidades de amostragem ${ }^{1}$
}

\author{
Vilmar A. Ragagnin², Darly G. de Sena Júnior ${ }^{2}$ \& Américo N. da Silveira Neto ${ }^{2}$
}

\begin{abstract}
RESU MO
O bjetivou-se, com este trabalho, estudar a variabilidade e a dependência espacial de variáveis de solo e 0 efeito sobre a recomendação de corretivo de três intensidades simuladas de amostragem. Em uma área de 725,9 ha coletaram-se 154 amostras compostas a uma distância de aproximadamente $225 \mathrm{~m}$ em uma malha regular. Dentro da faixa de dependência espacial foram eliminadas amostras e criados dois outros conjuntos de dados, com 76 e 38 amostras cada um. Realizou-se análise geoestatística das variáveis capacidade de troca catiônica e saturação por bases. A pós a interpolação por krigagem das variáveis se elaboraram os mapas de recomendação de calcário para os três conjuntos de dados. Em relação ao método convencional de recomendação não houve, pela média, redução na necessidade de calcário. Verificaram-se, então, diferenças na recomendação com as três intensidades de amostragem especialmente na distribuição espacial das doses; entretanto, mesmo considerando correta a recomendação com maior número de amostras, pela redução de custos e diferença considerada aceitável entre as recomendações, é viável a utilização da menor intensidade de amostragem.
\end{abstract}

Palavras-chave: variabilidade espacial, geoestatística, agricultura de precisão

\section{Recommendation of liming at variable rates under different sampling intensities}

\begin{abstract}
The aim of this work was to study the variability and spatial dependence of soil properties and their effects on lime recommendation with three simulated sampling intensities. In an area of $725.9 \mathrm{ha}, 154$ samples were collected at a distance of $225 \mathrm{~m}$ in a regular grid. Samples were discarded within the spatial dependence range and two other data sets were created, with 76 and 38 samples each. Geostatistical analyses were performed for the variables, cation exchange capacity and sum of bases. After kriging interpolation of the variables lime recommendation maps were elaborated for the three data sets. In comparison with the traditional recommendation method, on average, there was no reduction on lime requirement. Differences among the three recommendation rates were noted, specially on spatial distribution of doses. On the other hand, assuming as correct recommendation with higher number of samples, the use of a lower intensity sampling strategy is viable due to the cost reduction and acceptable difference among recommendations.
\end{abstract}

Key words: spatial variability, geostatistics, precision agriculture

1 Projeto financiado pelo Programa de Apoio a Projetos Institucionais do CAJ PROAPI/CAJ-UFG

2 UFG, Campus Jataí, Rod. BR 364, km 192, CP 3, CEP 75800-000, Jataí, GO . Fone: (64) 3632-2101; 3632-1510. E-mail: vilmar.ragagnin@gmail.com; darly.sena@gmail.com; americonunesagro@yahoo.com.br 


\section{INTRODUÇÃO}

A implementação da Agricultura de Precisão (AP) pode contribuir para otimização da produção agrícola e redução do risco de contaminação ambiental. Um componente fundamental da AP é a medição da variabilidade espacial nas características do solo e sua influência na variabilidade da cultura (Earl et al., 2003). Em geral, a expectativa é o aumento de produtividade e uniformização das áreas agrícolas, mas se pode explorar o máximo potencial produtivo da área agrícola, fato que levaria ao uso de maiores doses nas parcelas com maior potencial, para maximizar a produção e/ou repor nutrientes exportados pela cultura.

A área de fertilidade do solo é a que envolve maiores custos e dificuldades para identificação da variabilidade espacial (Godwin \& Miller, 2003). Embora sensores já estejam sendo empregados para o mapeamento das propriedades do solo, têm sido utilizados, atualmente, dois métodos principais: amostragem em malha ou em zonas de manejo. Quando se utiliza amostragem em malha são empregados, também, métodos de interpolação, como krigagem, inverso da distância ponderada e outros, para a geração dos mapas (Schloeder et al., 2001). A krigagem é o método que proporciona maior exatidão na estimativa dos valores em pontos não amostrados e maior precisão na elaboração dos mapas (Corá \& Beraldo, 2006). Para tanto se utilizam técnicas de geoestatística para análise da estrutura espacial dos atributos do solo (Isaaks \& Srivastava, 1989) e verificação da ocorrência de dependência espacial entre as amostras, medida pelos variogramas (Gomes et al., 2007b). Entretanto, uma densa malha de observações é necessária para a construção dos variogramas e, além disso, a exatidão tende a aumentar com o tamanho da amostra (Schloeder et al., 2001). A qualidade das estimativas pode ser avaliada por diversos métodos, mas, em geral, os melhores resultados têm sido obtidos pela avaliação dos resultados da validação cruzada (Robinson \& Metternicht, 2006; Carvalho et al., 2009).

Uma estratégia bem feita de amostragem de solos é fundamental, apesar da dificuldade para se estabelecer a distância entre amostras e o número de amostras ideal. Devem ser atendidos, ao mesmo tempo, requisitos técnicos e econômicos, para identificar e compreender a variabilidade dos diferentes atributos de solo; se a distância entre amostras for superior àquela em que há correlação espacial, os resultados da interpolação não serão confiáveis; por outro lado, a amostragem de solos muito intensa pode inviabilizar economicamente a análise das características físicas e químicas do solo. Visa-se com esse trabalho simular diferentes intensidades de amostragem para a Capacidade de Troca Catiônica (CTC) e Saturação por Bases (V) a partir de dados coletados em amostragem em malha e avaliar seus efeitos sobre a recomendação de corretivo a taxa variada.

\section{MATERIAL E MÉTODOS}

Neste trabalho foram utilizados dados da Fazenda Paraíso, localizada no município de Jataí, GO, que tem sido cul- tivada com soja na safra e com milho em 'safrinha', em um sistema de cultivo mínimo. Em uma área de 725,9 ha, submetida dois anos antes da coleta dos dados à calagem em taxa variável, obtiveram-se 154 amostras compostas a partir de 10 amostras simples coletadas em um raio de $20 \mathrm{~m}$. As amostras foram georreferenciadas com GPS no ponto central de amostragem, distanciadas cerca de $225 \mathrm{~m}$ em uma malha regular. A partir das análises de solo procedeu-se à análise geoestatística utilizando-se a versão de demonstração do programa computacional GS+. Obteve-se, então, a semivariância para todos os pares de pontos possíveis, para avaliação da dependência espacial entre amostras e se ajustou uma função aos dados para as variáveis, isoladamente. Foram utilizados os modelos esférico, exponencial, gaussiano, linear e linear com patamar.

Os modelos foram submetidos a validação cruzada, em que cada ponto amostrado é excluído do banco de dados e seu valor é estimado através do modelo ajustado. O procedimento é repetido para todos os pontos e realizado o ajuste de uma regressão linear simples entre os valores observados e os estimados. O modelo escolhido foi aquele no qual o intercepto da regressão foi mais próximo de zero e a inclinação da reta mais se aproximou de um. Os parâmetros para ajuste dos modelos foram definidos por tentativa e análise do resultado da validação cruzada. Os semivariogramas do conjunto de dados original foram analisados para verificar a existência de dependência espacial nas variáveis estudadas e determinar a distância a partir da qual as amostras passaram a ser independentes (alcance).

A fim de avaliar o efeito da coleta de número menor de amostras visando à redução dos custos a partir da grade original de 154 pontos (Figura 1A) foram simuladas outras duas intensidades de amostragem; com isto, foram criados dois novos bancos de dados, com 76 (Figura 1B) e 38 pontos (Figura 1C), por meio da exclusão de amostras intermediárias produzindo uma malha regular mais esparsa. A exclusão das amostras respeitou o alcance definido pelos variogramas ajustados aos dados completos, mantendo a amostragem dentro da faixa de dependência espacial para as variáveis CTC e V. Esses novos bancos de dados foram submetidos às análises geoestatísticas para as mesmas variáveis. A escolha dos modelos e parâmetros para ajuste dos variogramas foi feita com base nos mesmos critérios utilizados para o conjunto de dados completo.

Os modelos escolhidos foram utilizados para interpolação por meio de krigagem no programa computacional GS+. Utilizou-se o método de interpolação em blocos criando-se células de $40 \times 40 \mathrm{~m}$, área próxima à utilizada no campo para a coleta das amostras de solo. Utilizaram-se os dados interpolados das duas variáveis com vista à para elaboração dos mapas de recomendação de calagem em um Sistema de Informações Geográficas (SIG). Utilizou-se o critério de elevar a saturação por bases a 50\% considerando-se um calcário com PRNT igual a 100. Calculou-se o valor médio das duas variáveis nas 154 amostras e, com esses resultados, se calculou a dose recomendada pela média geral da área. Para comparação entre os mapas de recomendação elaborados com as três intensidades de amostragem utilizou-se, como referência, a recomendação, a partir das 154 amostras. A similaridade foi inferida pela subtração 
A.

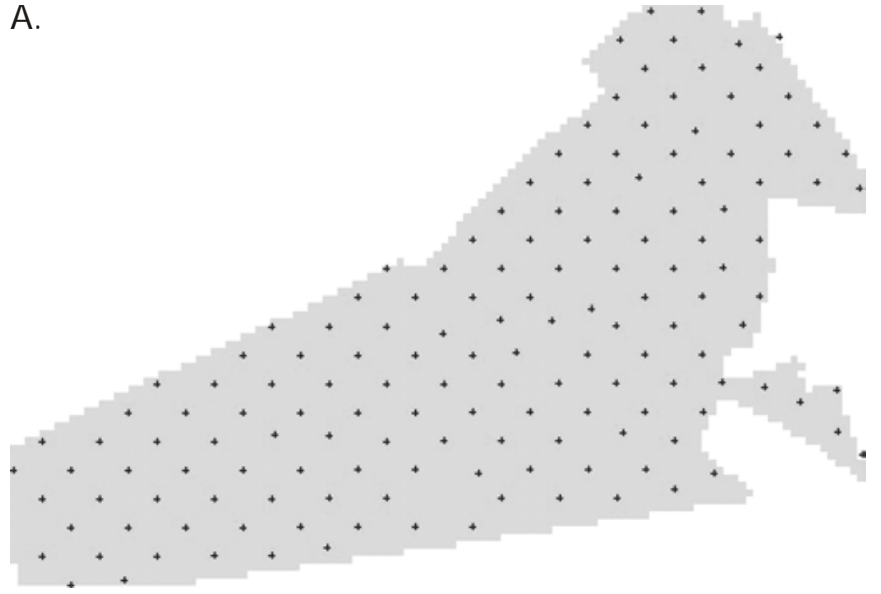

B.

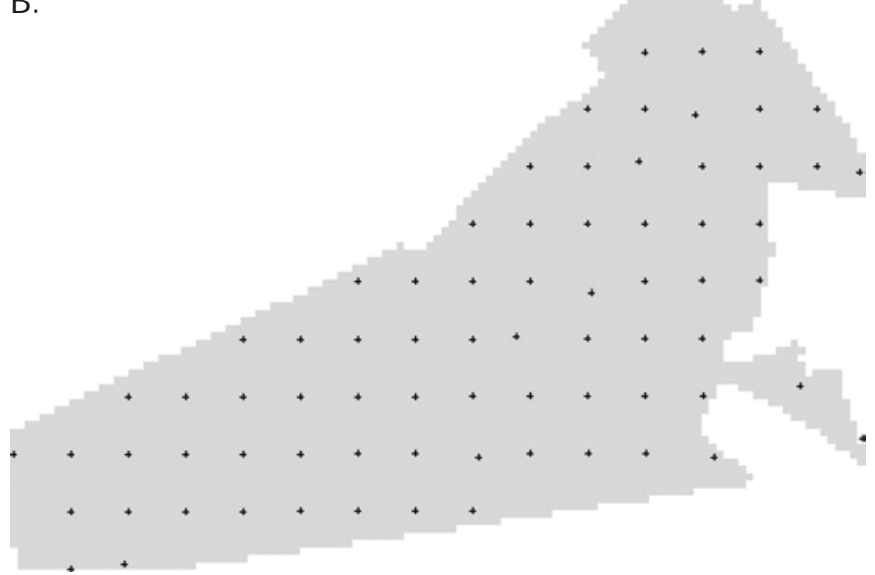

C.

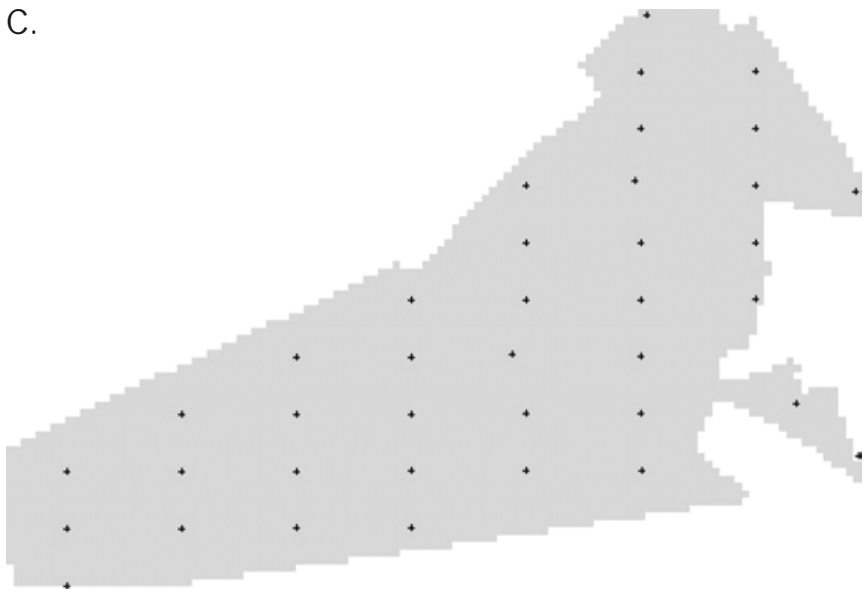

Figura 1. Mapas representando os três conjuntos de dados: amostragem completa 154 pontos (A), e bancos de dados com 76 (B) e 38 pontos (C)

entre os mapas gerados e pela correlação linear entre as recomendações para as mesmas células.

\section{RESULTADOS E DISCUSSÃO}

O modelo de maior ocorrência para os atributos do solo, segundo Bertolani \& Vieira (2001) é o esférico, mas Gomes et al. (2007a) não verificaram predominância entre os modelos esférico e exponencial no estudo da variabilidade espacial de atributos físicos do solo. No presente trabalho o modelo esférico foi o melhor apenas para a variável CTC, com o conjunto de dados completo (Figura 2A). Com os dois outros conjuntos de dados para a mesma variável, o modelo selecionado foi exponencial, tanto para 76 (Figura 2B) como 38 pontos (Figura $2 \mathrm{C}$ ). Para a variável V o modelo que apresentou melhores resultados da validação cruzada foi o gaussiano nas três intensidades de amostragem (Figuras 3A, B e C). As estimativas dos parâmetros dos modelos ajustados são apresentadas na Tabela 1.

Verificou-se que as variáveis estudadas apresentaram razão do efeito pepita em relação ao patamar, menor que $25 \%$, indicando forte dependência espacial nas três intensidades de amostragem, de acordo com critérios estabelecidos por Cambardela et al. (1994). O parâmetro efeito pepita mostra a variabilidade não explicada que, nesse estudo, apresentou valores reduzidos, cujo alcance indica o limite da dependência

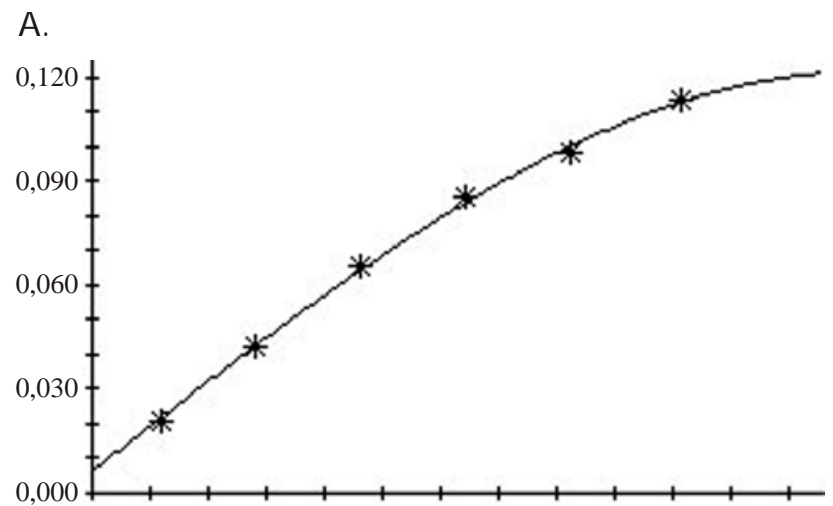

B.

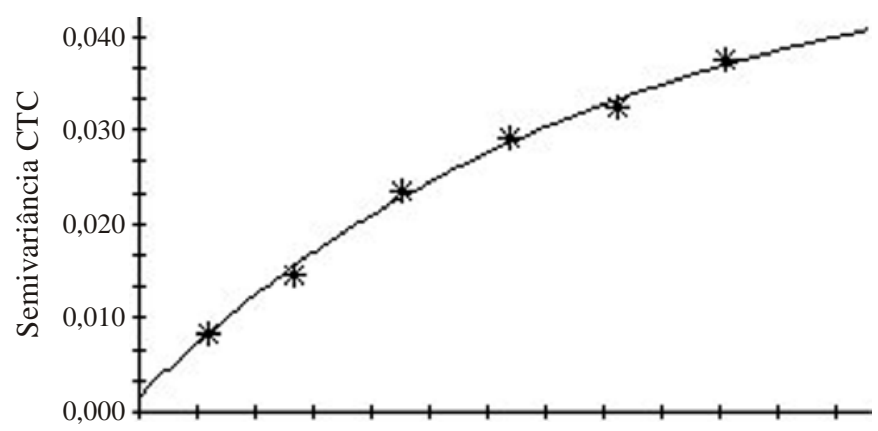

C.

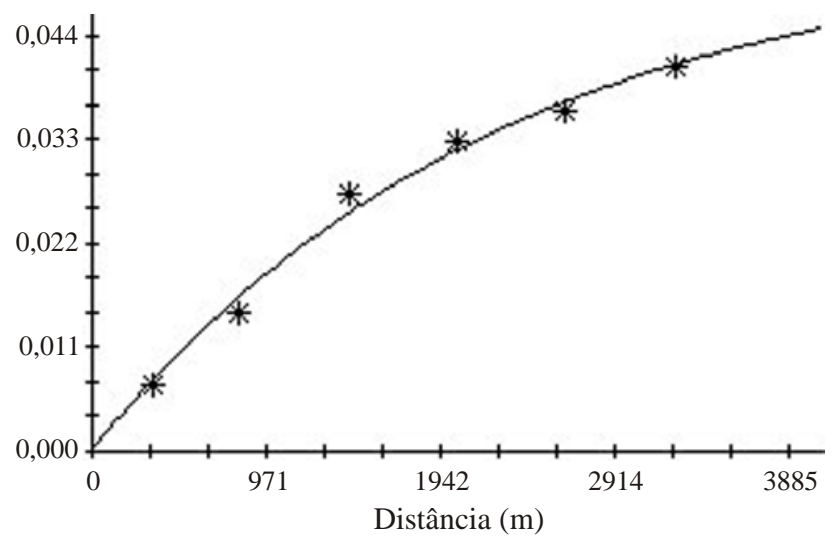

Figura 2. Semivariogramas ajustados para Capacidade de Troca Catiônica (CTC) com três intensidades de amostragem: 154 (A), 76 (B) e 38 pontos (C) 
A.

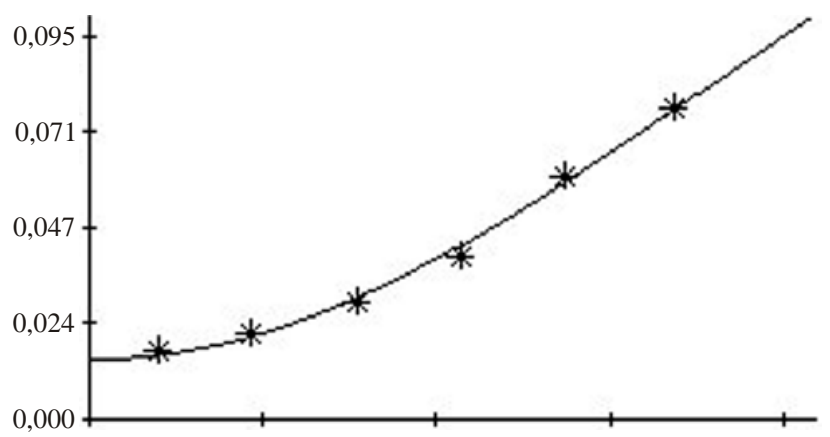

B.

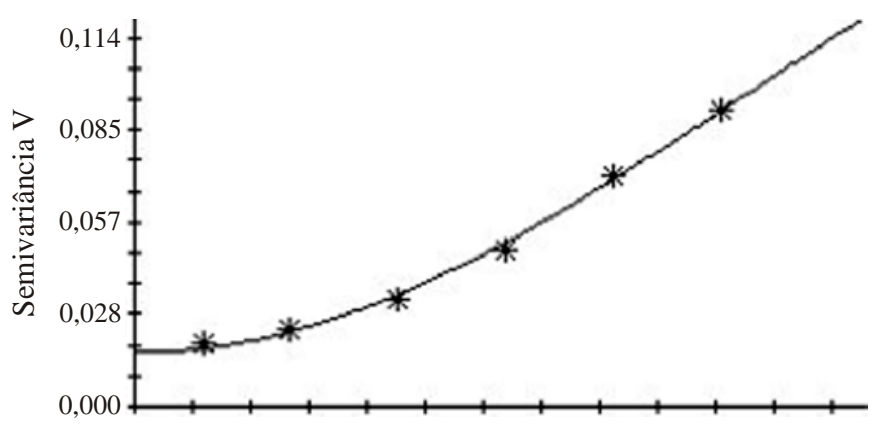

C.

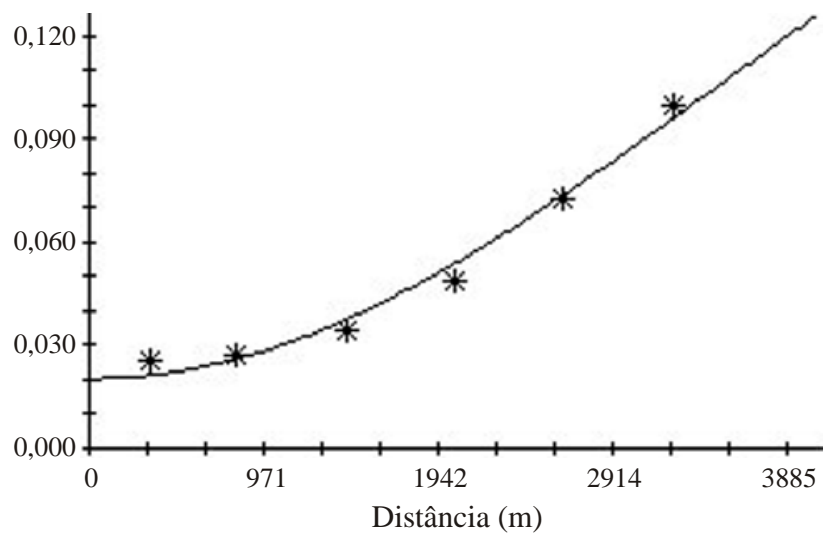

Figura 3. Semivariogramas ajustados para Saturação por Bases (V) com três intensidades de amostragem: $154(A), 76$ (B) e 38 pontos (C)

espacial da variável. Neste estudo o alcance da dependência espacial da variável CTC apresentou valor de $4.272 \mathrm{~m}$ no conjunto de dados completo. Com os conjuntos de dados reduzidos para 76 e 38 pontos encontraram-se valores de alcance de 2.620 e $2.252 \mathrm{~m}$, respectivamente. Houve redução do alcance com a redução da intensidade da amostragem chegando a $52 \%$ do valor observado no conjunto de dados completo quando se trabalhou com 38 amostras; mesmo assim, nos três conjuntos de dados a amostragem permaneceu dentro da faixa de dependência espacial e o menor valor, $2.252 \mathrm{~m}$, ainda é superior ao utilizado em muitas situações com amostragem a cada 4 ou 5 ha; por outro lado, o alcance para a variável V praticamente não foi alterado pela redução no número de amostras, com os valores variando entre 4.848 a $5.001 \mathrm{~m}$.

Realizou-se a qualidade da interpolação dos dados por meio do coeficiente da regressão e intercepto da validação cruzada. Em uma situação ideal o coeficiente da regressão seria igual a um e o intercepto igual a zero. Tanto para a variável CTC (Figura 4A), como para a variável V (Figura 5A), com o conjunto de dados completo os resultados foram satisfatórios e, com os outros conjuntos de dados, embora houvesse menor número de pares de pontos disponíveis para ajuste dos modelos, não houve decréscimo na qualidade das estimativas da interpolação por meio da krigagem. Para a variável CTC, tanto com o conjunto de 76 pontos (Figura 4B) como com o conjunto de 38 pontos (Figura 4C), os resultados da validação cruzada foram próximos aos do conjunto de dados completo. Para a variável V ocorreu o mesmo comportamento na qualidade das estimativas para 76 pontos (Figura 5B) e 38 pontos (Figura 5C), em relação ao conjunto de dados completo. Em geral, as estimativas obtidas com pequeno número de amostras tendem a ser menos precisas mas, neste trabalho, não se observou tal efeito. Um dos fatores que podem ter contribuído para este resultado é o fato de ter sido respeitado o alcance das variáveis para definição da distância entre amostras.

Apresentam-se, na Tabela 2, o resumo das recomendações com os três conjuntos de dados e a dose recomendada pela média geral. Verifica-se que a dose média recomendada nos quatro casos foi semelhante porém com grande amplitude nos casos em que se utilizou a taxa variável. Ressalta-se que é uma situação pouco frequente na prática, haja vista que, mesmo para recomendação de calagem pela média, não se deve trabalhar com apenas uma amostra composta para uma área tão extensa. Cantarutti et al. (1999) recomendam, para glebas grandes, mesmo que homogêneas, a subdivisão em glebas de até 10 ha.

A quantidade total de calcário necessária foi superior quando se trabalhou a taxa variável com 154 e 76 pontos em relação ao sistema convencional. Em seu trabalho, Weirich Neto et al. (2006) observaram uma possível economia de 7,84 t de calcário em uma área de 9,6 ha em relação ao método convencional; entretanto, neste trabalho a variável Soma

Tabela 1. Parâmetros dos modelos dos semivariogramas ajustados para as características Capacidade de Troca Catiônica (CTC) e Saturação por Bases (V) do solo, em três intensidades de amostragem

\begin{tabular}{ccccccccc}
\hline Variável & Pontos & Modelo & Alcance $(\mathbf{m})$ & Efeito pepita & patamar & Pepitalpatamar & SQ Erros & $\mathbf{R}^{2}$ \\
& 154 & esférico & 4272 & 0,0023 & 0,0439 & 0,052 & $1,364 \mathrm{E}^{-6}$ & 0,998 \\
CTC & 76 & exponencial & 2620 & 0,0016 & 0,0508 & 0,031 & $2,77 \mathrm{E}^{-6}$ & 0,996 \\
& 38 & exponencial & 2252 & 0,0001 & 0,0534 & 0,002 & $9,84 \mathrm{E}^{-6}$ & 0,989 \\
\hline \multirow{2}{*}{ V } & 154 & gaussiano & 4889 & 0,0015 & 0,1861 & 0,080 & $1,344 \mathrm{E}^{-5}$ & 0,995 \\
& 76 & gaussiano & 4848 & 0,0166 & 0,2214 & 0,075 & $1,18 \mathrm{E}^{-5}$ & 0,997 \\
& 38 & gaussiano & 5001 & 0,0203 & 0,2406 & 0,084 & $6,47 \mathrm{E}^{-5}$ & 0,985 \\
\hline
\end{tabular}


A.

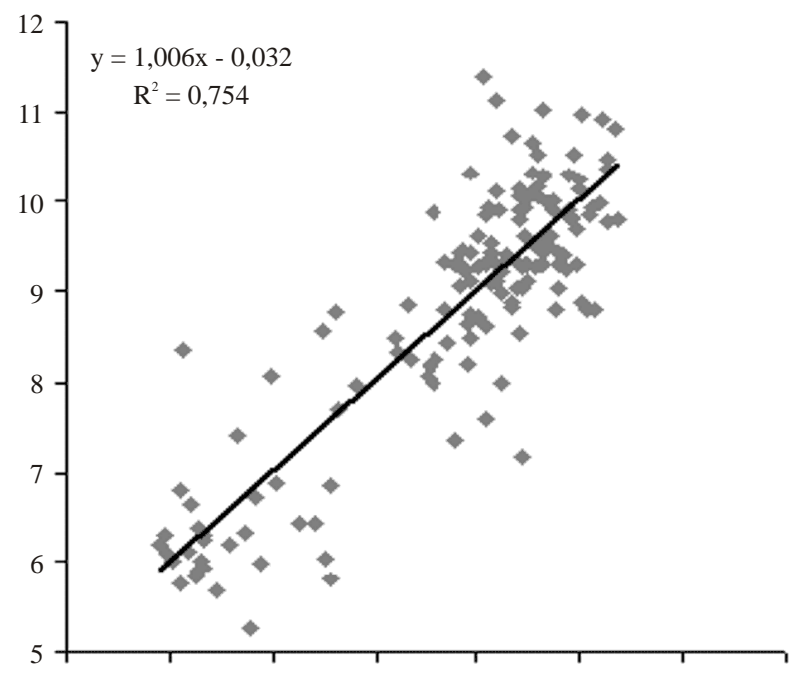

B.

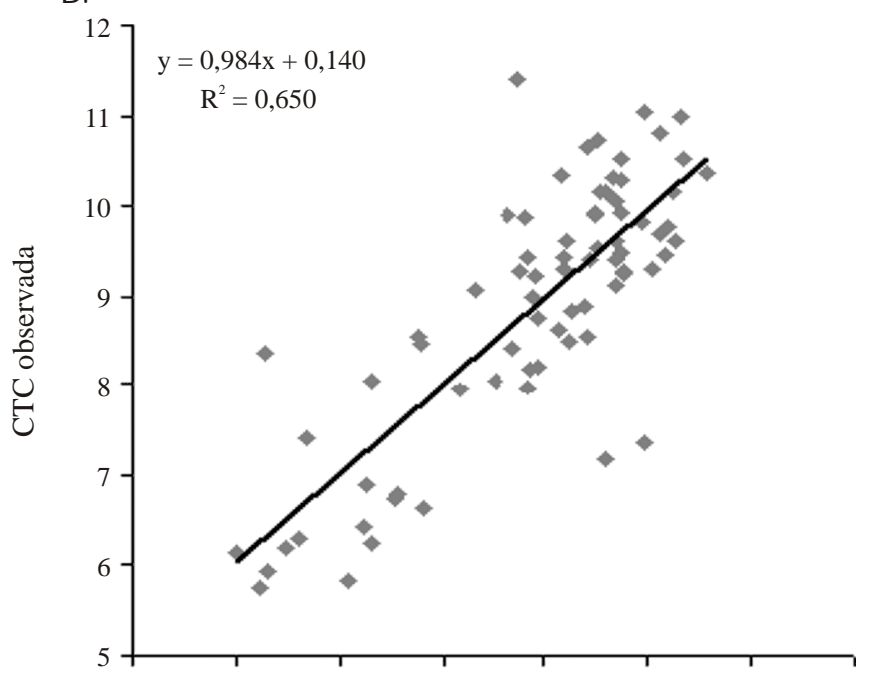

C.

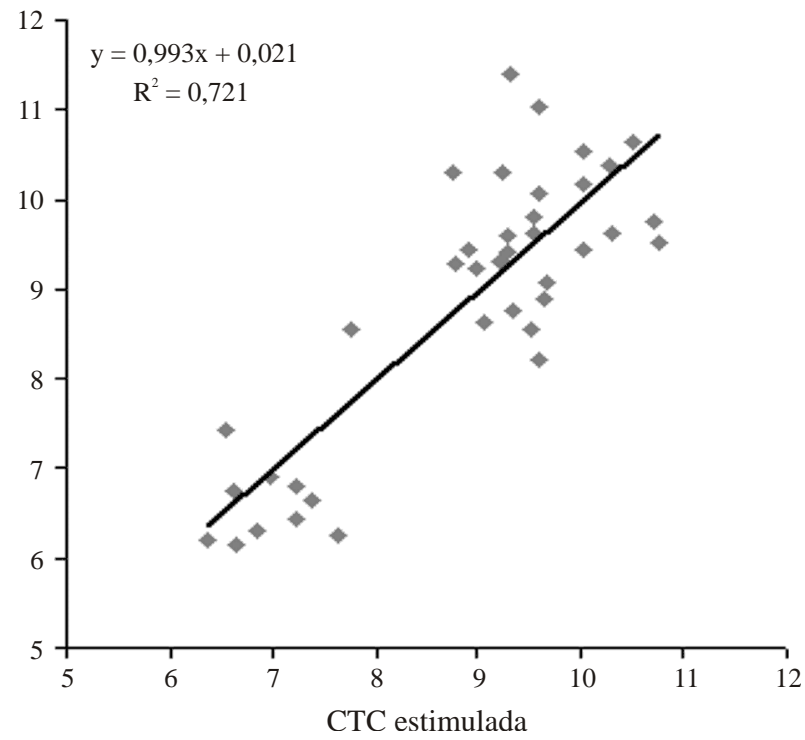

Figura 4. Gráficos da val idação cruzada para Capacidade de Troca Catiônica (CTC) com três conjuntos de dados: 154 (A), 76 (B) e 38 pontos (C)
A.

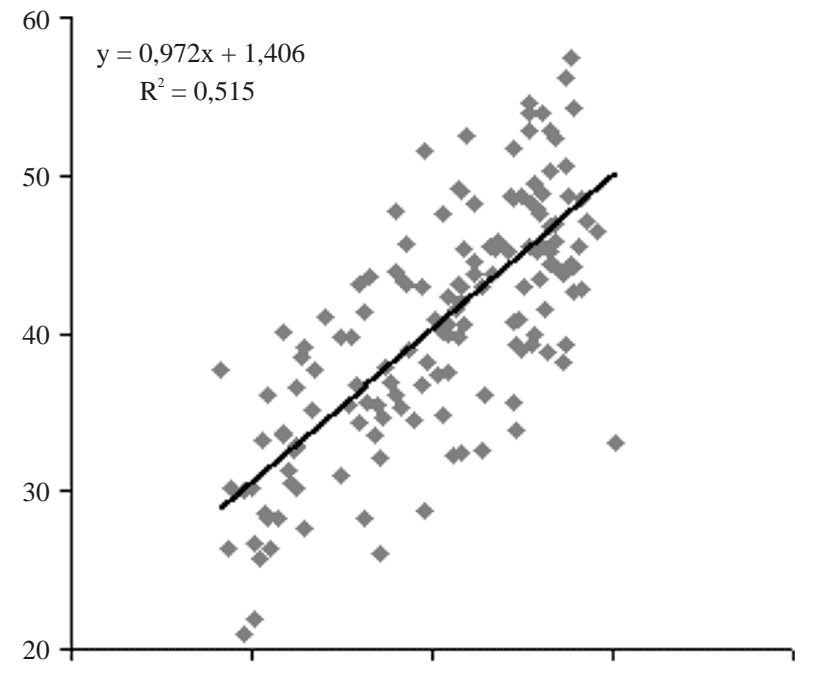

B.

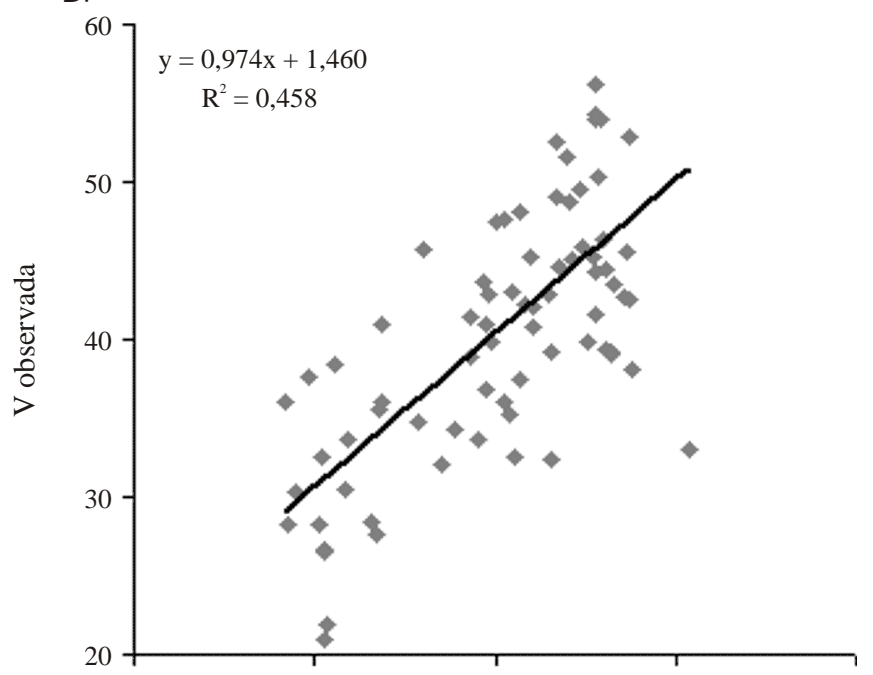

C.

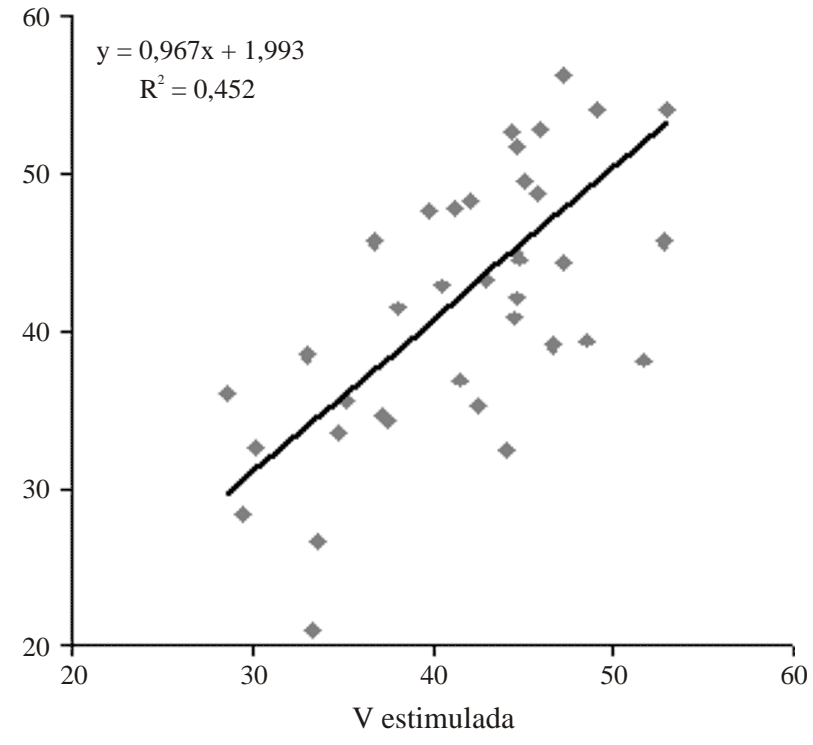

Figura 5. G ráficos da validação cruzada para Saturação por Bases $(V)$ com três conjuntos de dados: 154 (A), 76 (B) e 38 pontos (C) 
Tabela 2. Doses de calcário recomendadas e total necessário para aplicação a taxas variáveis e pelo método convencional, na área de 725,9 ha

\begin{tabular}{ccccccc}
\hline & & \multicolumn{4}{c}{ Dose (t ha-1) } & \\
\cline { 3 - 6 } Método & Amostras & Mín. & Máx. & Média & $\begin{array}{c}\text { Desvio } \\
\text { Padrão }\end{array}$ & \\
& 154 & 0 & 2,084 & 0,886 & 0,554 & 642,8 \\
Taxa & $\mathbf{t}$ ) \\
variável & 76 & 0,081 & 1,941 & 0,874 & 0,564 & 634,7 \\
& 38 & 0 & 2,005 & 0,805 & 0,595 & 579,4 \\
\hline Média & 1 & - & - & 0,816 & - & 592,2 \\
\hline
\end{tabular}

de Bases não apresentou dependência espacial de modo que o mapa de necessidade de calagem foi obtido pelo método do vizinho mais próximo. Por sua vez, Corá \& Beraldo (2006) constataram que a quantidade total de calcário calculada considerando-se os valores médios ou os valores específicos de cada local, foi semelhante.

No presente trabalho, se utilizada a dose média geral recomendada $\left(0,816 \mathrm{t} \mathrm{ha}^{-1}\right)$ apenas $15 \%$ da área (109,3 ha) receberiam a dose correta com a amostragem de 154 pontos. Oliveira et al. (2008) verificaram redução no total de corretivo na cultura do café com a utilização de taxas variadas porém no primeiro ano $61,95 \%$ da área receberiam dose superior à recomendada pela média, enquanto no ano seguinte $75,52 \%$ da área receberiam dose inferior. Na Figura 6A se apresenta

A.

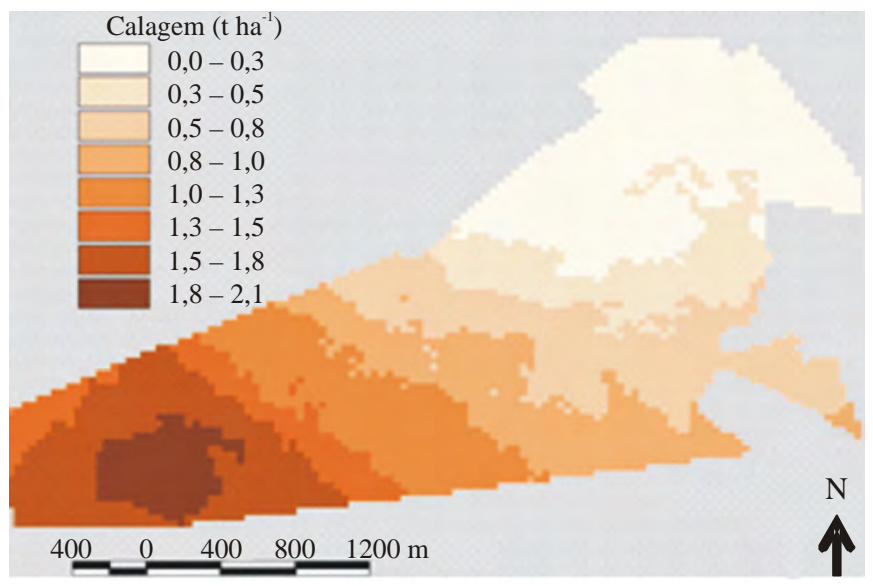

B.

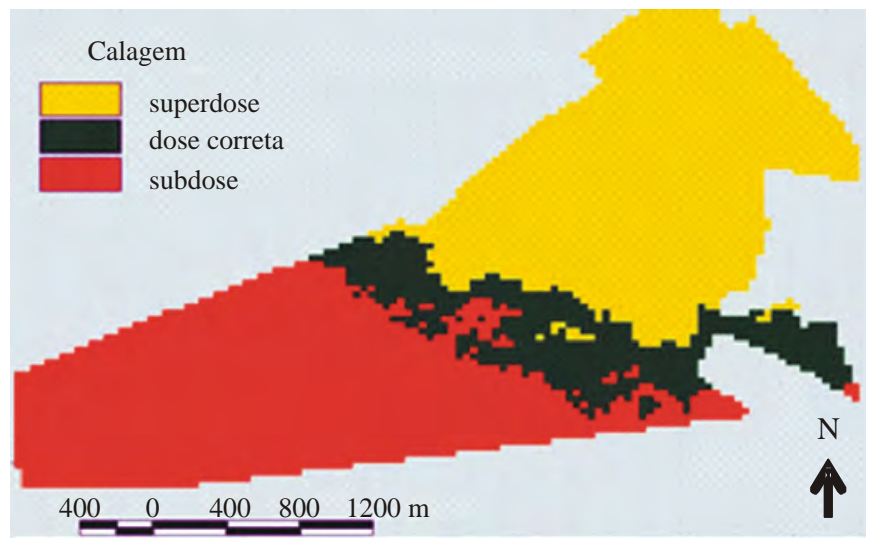

Figura 6. Mapa de recomendação de calagem a taxa variada a partir das 154 amostras (A) e a área com dose correta se utilizada a dose média geral para a área (B) o mapa de recomendação de calagem a taxa variada, a partir do conjunto de dados completo; já na Figura 6B se acha o mapa indicando as áreas nas quais seriam aplicadas a dose correta, a superdose e a subdose, caso a calagem fosse recomendada pela média. Consideraram-se corretas as recomendações para as células cujas doses diferiram da média em até $0,1 \mathrm{t} \mathrm{ha}^{-1}$ para mais ou para menos $\left(0,716\right.$ a $\left.0,916 \mathrm{t} \mathrm{ha}^{-1}\right)$.

Para efeito de comparação entre as recomendações fez-se a subtração entre os mapas de recomendação. As diferenças resultantes foram agrupadas em classes com amplitude de $0,1 \mathrm{t} \mathrm{ha}^{-1}$. Nas classes $-0,1$ a 0 e 0 a $0,1 \mathrm{t} \mathrm{ha}^{-1}$ considerou-se que as recomendações eram iguais, representadas em branco; em azul estão representadas as áreas nas quais seria aplicada dose de calcário maior e, em vermelho, dose menor, se fosse reduzido o número de amostras em relação às 154 originais. Na Figura 7 está representada a diferença entre a recomendação do conjunto de dados completo e de 76 (Figura 7A) e de 38 pontos (Figura 7B).

Na Fazenda em estudo se utiliza o critério prático de não aplicar o corretivo quando a dose recomendada é inferior a $0,5 \mathrm{t} \mathrm{ha}^{-1}$; desta forma, a necessidade total de calcário seria de 578,9, 570,9 e 528,0 t para os conjuntos de dados de 154, 76 e 38 pontos, respectivamente, enquanto com a aplicação pela média seria necessário aplicar 592,2 t. O uso de critéri-

A.

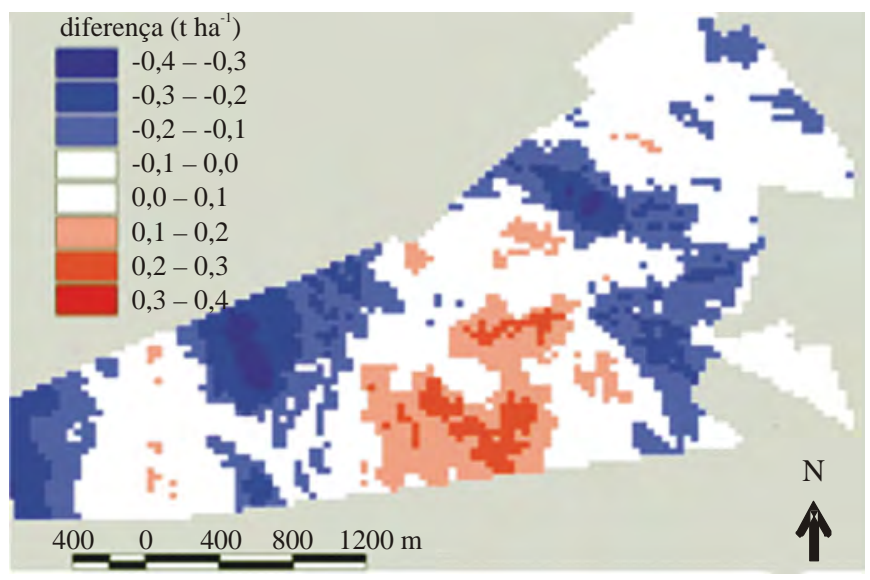

B.

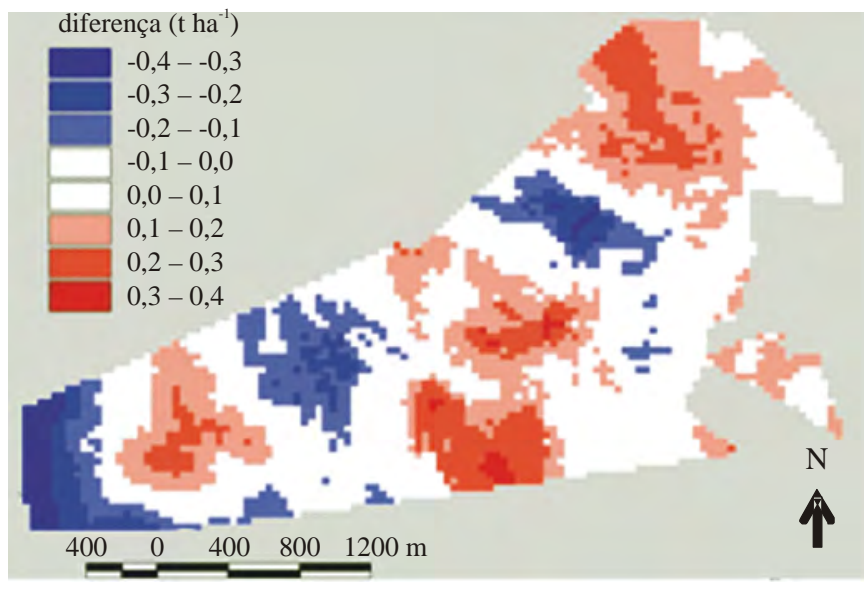

Figura 7. Mapas de diferença entre recomendações com 76 pontos (A) e 38 pontos (B) em relação ao conjunto de dados completo 
os semelhantes talvez seja o motivo da redução na necessidade de insumos quando se utiliza a aplicação a taxa variada. Com a utilização deste critério a diferença entre as recomendações foi ainda menor quando se alterou o número de pontos (Tabela 3). A diferença entre a recomendação do conjunto de dados completo e de 76 e de 38 pontos, quando não se aplicam doses menores que $0,5 \mathrm{t} \mathrm{ha}^{-1}$, está ilustrada nas Figuras 8A e B, respectivamente.

Tabela 3. Diferença percentual da área de aplicação com recomendação obtida com conjunto de dados de 154 pontos em relação à área total ou nas áreas com recomendação acima de 0,5 tha-1

\begin{tabular}{ccccccc}
\hline \multirow{2}{*}{ classes } & \multicolumn{2}{c}{$\mathbf{7 6}$ amostras } & & \multicolumn{2}{c}{$\mathbf{3 8 \text { amostras }}$} \\
\cline { 2 - 3 } \cline { 5 - 6 } & Toda área & $\mathbf{0} \mathbf{0 , 5} \mathbf{t ~ h a}^{-1}$ & & Toda área & $\mathbf{0 , 5}$ t ha-1 $^{-1}$ \\
$-0,4$ a $-0,3$ & 1,21 & 1,08 & & 1,13 & 1,11 \\
$-0,3$ a $-0,2$ & 4,58 & 4,06 & & 2,51 & 1,69 \\
$-0,2$ a $-0,1$ & 14,00 & 10,40 & & 6,29 & 4,87 \\
$-0,1$ a 0,1 & 51,22 & 61,74 & & 44,72 & 64,39 \\
0,1 a 0,2 & 17,79 & 12,08 & & 27,50 & 16,62 \\
0,2 a 0,3 & 8,62 & 8,13 & & 15,95 & 9,51 \\
0,3 a 0,4 & 2,58 & 2,51 & & 1,89 & 1,82 \\
\hline
\end{tabular}

A.

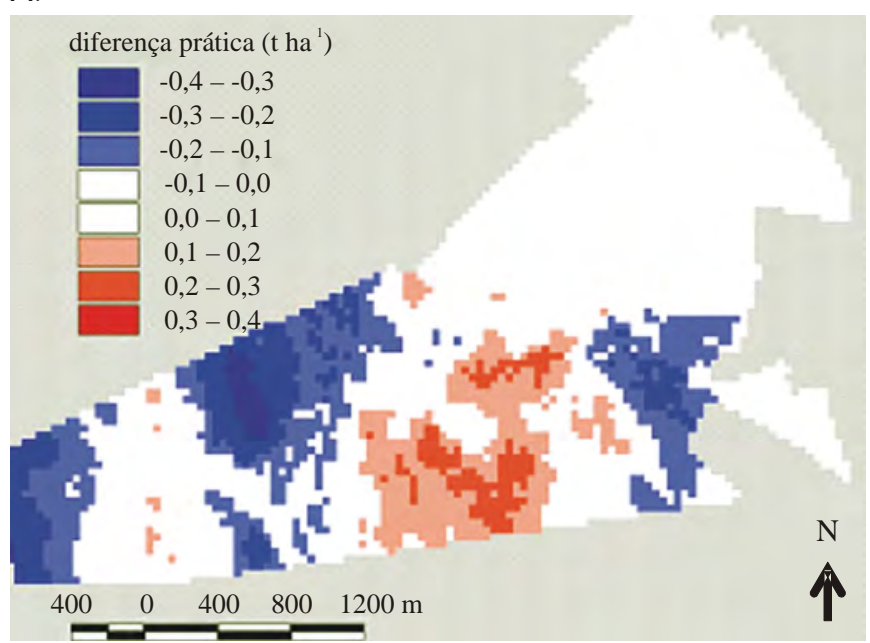

B.

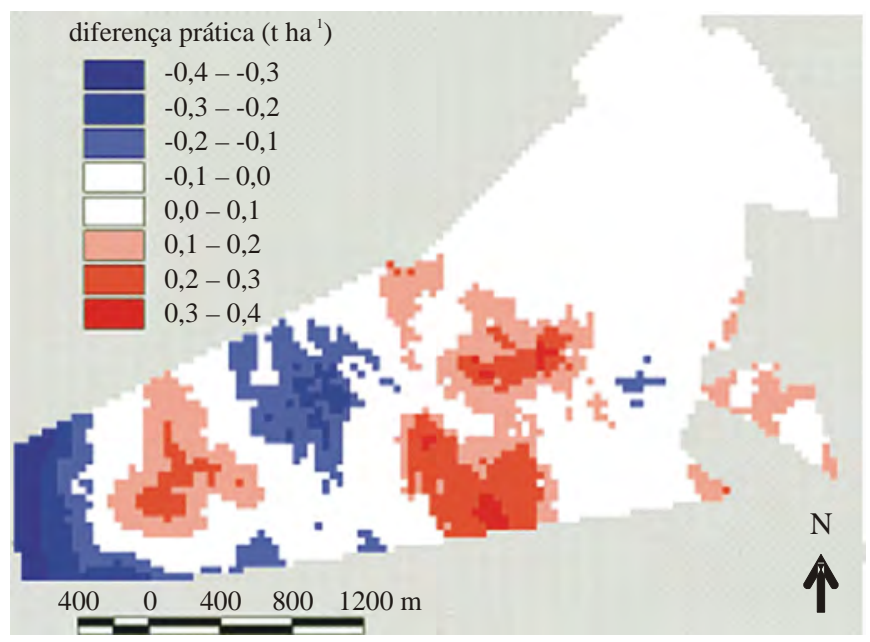

Figura 8. Mapas de diferença entre recomendação com 76 pontos $(A)$ e 38 pontos (B) em relação ao conjunto de dados completo, aplicando-se somente doses acima de $0,5 \mathrm{t} \mathrm{ha}^{-1}$

\section{CONCLUSÕES}

1. As estimativas da interpolação por krigagem não foram prejudicadas pelo maior espaçamento e distância entre amostras, avaliadas pela validação cruzada.

2. A recomendação de calagem em taxas variadas na área em estudo não reduziu a quantidade total de calcário em relação ao método convencional de recomendação, a não ser que se utilize o critério prático de não se aplicar doses inferiores a $0,5 \mathrm{tha}^{-1}$.

3. A redução da intensidade de amostragem, respeitandose a faixa de dependência espacial, provocou diferenças na distribuição espacial da recomendação de calagem e na quantidade total de calcário necessária.

4. Em função da redução nos custos e pela diferença entre as recomendações, considerou-se viável a redução da intensidade de amostragem.

\section{LITERATURA CITADA}

Bertolani, F. C.; Vieira, S. R. Variabilidade espacial da taxa de infiltração de água e da espessura do horizonte $\mathrm{A}$, em um Argissolo Vermelho-Amarelo, sob diferentes usos. Revista Brasileira Ciência do Solo, v.25, n.4, p.987-995, 2001.

Cambardella, C. A.; Moorman, T. B.; Novak, J. M.; Parkin, T. B.; Karlen, D. L.; Turco, R. F.; Konopka, A. E. Field-scale variability of soil properties in central Iowa soils. Soil Science Society of America Journal, v.58, n.5, p.1501-1511, 1994.

Cantarutti, R. B.; Alvarez V., V. H.; Ribeiro, A. C. Amostragem do solo In: Ribeiro, A. C.; Guimarães, P. T.; Alvarez, V. V. H. (ed.) Recomendações para o uso de corretivos e fertilizantes em Minas Gerais, $5^{\mathrm{a}}$ aproximação. Viçosa: CFSEMG, 1999. Cap. 3, p.13-20.

Carvalho, J. R. P.; Vieira, S. R.; Grego, C. R. Comparação de métodos para ajuste de modelos de semivariograma da precipitação pluvial anual. Revista Brasileira de Engenharia Agrícola e Ambiental, v.13, n.4, p.443-448, 2009.

Corá, J. E.; Beraldo, J. M. G. Variabilidade espacial de atributos do solo antes e após calagem e fosfatagem em doses variadas na cultura de cana-de-açúcar. Engenharia Agrícola, v.26, n.2, p.374-387, 2006.

Earl, R.; Taylor, J. C.; Wood, G. A.; Bradley, I.; James, L. T.; Waine, T.; Welsh, J. P. Godwin, R. J.; Knight, S. M. Soil factors and their influence on within-field crop variability. Part I: Field observation of soil variation. Biosystems Engineering, v.4, n.84, p.425-440, 2003.

Godwin, R. J.; Miller, P. C. H. A review of the technologies for mapping within-field variability. Biosystems Engineering, v.4, n.84, p.393-407, 2003.

Gomes, N. M.; Faria, M. A.; Silva, A. M.; Mello, C. R.; Viola, M. R. Variabilidade espacial de atributos físicos do solo associados ao uso e ocupação da paisagem. Revista Brasileira de Engenharia Agrícola e Ambiental, v.11, n.4, p.427-435, 2007 a.

Gomes, N. M.; Silva, A. M.; Mello, C. R.; Faria, M. A.; Oliveira, P. M. Métodos de ajuste e modelos de semivariograma aplicados ao estudo da variabilidade espacial de atributos físicohídricos do solo. Revista Brasileira de Ciência do Solo, v.31, n.3, p.435-443, $2007 \mathrm{~b}$. 
Isaaks, H. E.; Srivastava, R. M. A. Introduction to applied geoestatistics. Oxford: Oxford University Press. 1989. 560p.

Oliveira, R. B.; Lima, J. S. S.; Xavier, A. C.; Passos, R. R.; Silva, S. A.; Silva, A. F. Comparação entre métodos de amostragem do solo para recomendação de calagem e adubação do cafeeiro conilon. Engenharia Agrícola, v.28, n.1. p.176-186, 2008.

Robinson, T. P.; Metternicht, G. Testing the performance of spatial interpolation techniques for mapping soil properties. Computers and Electronics in Agriculture, v.50, n.2, p.97-108, 2006.
Schloeder, C. A.; Zimmerman, N. E.; Jacobs, M. J. Comparison of methods for interpolating soil properties using limited data. Soil Science Society of America Journal, v.65, n.2, p.470-479, 2001.

Weirich Neto, P. H.; Sverzut, C. B.; Schimandeiro, A. Necessidade de fertilizante e calcário em área sob sistema plantio direto considerando variabilidade espacial. Revista Brasileira de Engenharia Agrícola e Ambiental, v.10, n.2, p.338-343, 2006. 